\title{
RETRACTING DIFFEOMORPHISMS OF $n$-SPHERES
}

\section{J. M. ROBERTSON}

1. Introduction. Connectedness properties of various spaces of orientation preserving homeomorphisms of the $n$-sphere $S^{n}$ onto itself have been studied for some time. If $K_{n}, K_{n}^{\prime}, K_{n}^{\prime \prime}$ are the spaces of orientation preserving homeomorphisms with uniform convergence topology, $C^{\infty}$ orientation preserving homeomorphisms (not requiring differentiable inverses) with $C^{k}$ topology and $C^{\infty}$ orientation preserving diffeomorphisms with $C^{k}$ topology respectively then the following results are known: the rotation group of $S^{2}$ is a strong deformation retract of $K_{2}$ (see [3]), the rotation group of $S^{2}$ is a strong deformation retract of $K_{2}^{\prime \prime}$ (see [7]) and $K_{n}^{\prime}$ is arcwise connected (see [6]). There are maps in $K_{6}^{\prime \prime}$ not differentiably isotopic to the identity (see [4]). $K_{2}$ and $K_{3}$ are locally arcwise connected (see [5]), and if $K_{3}^{\prime \prime}$ and the rotation group on $S^{3}$ are homotopically equivalent then $K_{3}$ and the rotation group on $S^{3}$ are also (see [2]).

If one replaces the $C^{k}$ topology in $K_{n}^{\prime \prime}$ by its coarser pointwise counterpart where arcs are more plentiful, then the space has very nice connectedness properties. In particular the purpose of this paper is to prove the following:

THEOREM. The space of all $C^{\infty}$ orientation preserving diffeomorphisms of $S^{n}$ onto itself under the pointwise $C^{k}$ topology has as a strong deformation retract the rotation group of $S^{n}$.

Necessary mappings are constructed.

2. Pointwise $C^{k}$ topology and the spaces $H_{n}^{\prime \prime \prime}$. Let $D_{n}$ be the closed unit disk in $E_{n}$ and $H_{n}^{\prime \prime}$ be the space of $C^{\infty}$ diffeomorphisms of $D_{n}$ onto itself which are the identity in a neighborhood of the boundary under the $C^{k}$ topology. Let $H_{n}^{\prime \prime \prime}$ be the same set of functions under the pointwise $C^{k}$ topology. Convergence in $H_{n}^{\prime \prime}$ and $K_{n}^{\prime \prime}$ requires uniform convergence through the first $k$ derivatives while convergence in $H_{n}^{\prime \prime \prime}$ and $K_{n}^{\prime \prime}$ only requires pointwise convergence through those $k$ derivatives. A base of neighborhoods of a function $f$ consists of sets of functions whose first $k$ derivatives differs from those of $f$ by less than $\epsilon>0$ at a finite set of points, $\epsilon$ and the finite set varying. In particular the $C^{k}$ topology is finer than the pointwise $C^{k}$ topology.

Lemma. $H_{n}^{\prime \prime \prime}$ is contractible to the identity map on $D_{n}$.

Received by the editors March 14, 1969. 
Proof. For $0 \leqq t \leqq 1$ let $D(t)$ be the $n$ disk given by $\left\{x=\left(x_{1}, x_{2}, \cdots, x_{n}\right) \in E^{n}:|x-(-t / 2,0, \cdots, 0)| \leqq-(3 t / 4)+1\right\}$.

Let $T(t)$ be the obvious mapping of $D(t)$ onto $D_{n}$ defined by $T\left(x_{1}, \cdots, x_{n}\right)=(4 /(-3 t+4))\left(x_{1}+t / 2, x_{2}, \cdots, x_{n}\right)$. Now for $f \in H_{n}^{\prime \prime \prime}$ and for $0 \leqq t \leqq 1$ define $f_{t}: D_{n} \rightarrow D_{n}$ by:

$$
\begin{aligned}
f_{t}(x) & =x \quad \text { if } x \in D(t), \\
& =T^{-1} f(T x) \quad \text { if } x \in D(t) .
\end{aligned}
$$

The mapping of $H_{n}^{\prime \prime \prime} X I \rightarrow H_{n}^{\prime \prime \prime}$ defined by $\theta_{1}(f, t)=f_{t}$ retracts $H_{n}^{\prime \prime}$ ' onto a subspace of $H_{n}^{\prime \prime \prime}$ such that if $h$ is in the subspace, then $h$ is the identity map in a neighborhood of the origin and of the boundary of $D_{n}$.

The Alexander mapping (see [1]) applied to this subspace shows $H_{n}^{\prime \prime \prime}$ is contractible to the identity map. In particular the Alexander mapping $\theta_{2}: H_{n}^{\prime \prime \prime} X I \rightarrow H_{n}^{\prime \prime}$ ' is defined by $\theta_{2}(h, t)=h^{t}$ where

$$
\begin{aligned}
h^{t}(x) & =t h(x / t), \quad 0<t \leqq 1, \quad\left(h \text { extended to be the identity outside } D_{n}\right) \\
& =x, \quad t=0 .
\end{aligned}
$$

(Note that $\theta_{1}$ is just a sliding Alexander type map restricted to $\frac{1}{4}$ $\leqq t \leqq 1$.) Then the map $\theta: H_{n}^{\prime \prime \prime} X I \rightarrow H_{n}^{\prime \prime \prime}$ defined by

$$
\begin{aligned}
\theta(f, t) & =\theta_{1}(f, 2 t), \quad 0 \leqq t \leqq \frac{1}{2}, \\
& =\theta_{2}\left(\theta_{1}(f, 1),-2 t+2\right), \quad \frac{1}{2} \leqq t \leqq 1
\end{aligned}
$$

is the required contraction mapping.

$\theta$ is continuous in the $C^{k}$ topology except at $t=1$. Also given $\theta(f, 1)$ and a point $x$, if $x=0$ then all functions $\theta(g, t), g \in H_{n}^{\prime \prime \prime}, 0 \leqq t \leqq \frac{1}{2}$ have all derivatives equal to $\theta(f, 1)$ at $x$. If $x \neq 0$ then there is a neighborhood $N_{f}$ of $f$ and an interval $(\zeta, 1]$ such that $\theta(g, t)$ has derivatives equal to $\theta(f, 1)$ at $x$ if $g \in N_{f}$ and $t \in(\zeta, 1]$.

\section{Deforming $K_{n}^{\prime \prime \prime}$.}

THEOREM. The rotation group of $S^{n}$ is a strong deformation retract of $K_{n}^{\prime \prime \prime}$.

Proof. The necessary retraction mapping can be constructed from mappings given in paragraphs III and IV of [6].

Let $p=(0,0, \cdots,-1)$ on $S^{n}$ and $P:\left\{\left(x_{1}, \cdots, x_{n+1}\right) \in S_{n}: x_{n+1} \leqq 0\right\}$ $\rightarrow E_{n}$ be the projection map $P\left(x_{1}, \cdots, x_{n+1}\right)=\left(x_{1}, \cdots x_{n}\right)$. For $f \in K_{n}^{\prime \prime \prime}$ there is a rotation $r_{f}$ of $S^{n}$ such that $r_{f} f(p)=p$ and the Jacobian matrix of $\operatorname{Pr}_{f} f\left(P^{-1} x\right)$ at $x=0$ is triangular with positive 
diagonal elements. Using mappings of [6, paragraph III], the mapping $P r_{f} f P^{-1}$ can be deformed by a mapping $\left(P r_{f} f P^{-1}\right)_{t}, 0 \leqq t \leqq 1$ which at $t=1$ is the identity in a neighborhood $N_{f}$ of the origin in $E_{n}$ and agrees with $\left(P r_{f} f P^{-1}\right)_{0}=P r_{f} f P^{-1}$ outside a larger neighborhood $N$. Furthermore the size of $N$ can be fixed over all $f \in K_{n}^{\prime \prime \prime}$ with the deformation continous with respect to $f$ in $K_{n}^{\prime \prime \prime}$. We now construct the retraction map $\psi$ which deforms $K_{n}^{\prime \prime \prime}$ onto the rotation group of $S_{n}$.

Define $\psi_{1}(f, t)$ for $(f, t) \in K_{n}^{\prime \prime \prime} X I$ by

$$
\begin{aligned}
\psi_{1}(f, t)(x) & =r_{f}^{-1} P^{-1}\left(\operatorname{Pr}_{f} f^{-1}\right)_{t}(P(x)) \quad \text { if } P(x) \in N \\
& =f(x) \quad \text { if } P(x) \notin N .
\end{aligned}
$$

Then $\psi_{1}$ retracts $K_{n}^{\prime \prime \prime}$ onto a subspace $J_{n}^{\prime \prime \prime}$ such that if $g \in J_{n}^{\prime \prime \prime}$ then $r_{g} g$ is the identity in a neighborhood of $p$. Retracting $J_{n}^{\prime \prime \prime}$ onto the rotation group is now easy using the lemma. Specific details exactly parallel those given in [6, paragraph IV] or in [7].

\section{REFERENCES}

1. J. W. Alexander, On the deformation of an $n$-cell, Proc. Nat. Acad. Sci. U.S.A. 9 (1923), 406-407.

2. J. Cerf, Topologie de certains espaces de plongements, Bull. Soc. Math. France 89 (1961), 227-380. MR 25 \#3543.

3. H. Kneser, Die Deformationssätze der einfach zusammenhängenden Flächen, Math. Z. 25 (1926), 362-372.

4. J. W. Milnor, On manifolds homeomorphic to the 7-sphere, Ann. of Math. (2) 64 (1956), 399-405. MR 18, 498.

5. J. H. Roberts, Local arcwise connectivity in the space $H_{n}$ of homeomorphisms of $S^{n}$ onto itself, Summary of Lectures, Summer Institute on Set Theoretic Topology, Madison, Wis., 1955, p. 110.

6. J. M. Robertson, Some topological properties of certain spaces of differentiable homeomorphisms of disks and spheres, Pacific J. Math. 15 (1965), 1383-1389. MR 32 \#3042.

7. S. Smale, Diffeomorphisms of the 2-sphere, Proc. Amer. Math. Soc. 10 (1959), 621-626. MR 22 \#3004.

UNIVERSITY OF WASHINGTON 